\title{
Target selectivity in mRNA silencing
}

\author{
$\mathrm{N}$ Aronin \\ University of Massachusetts, Worcester, MA, USA
}

\begin{abstract}
Despite the excitement and promise of RNA interference in treating neurodegenerative disease, disease gene $m R N A$ might resist $m R N A$ silencing. Conventional siRNA design does not uniformly distinguish a mutant from a wild-type allele. CAG expansions in trinucleotide repeat diseases are unselective targets
\end{abstract}

for small siRNAs. This review will consider recent discoveries in mechanisms of RNA interference and siRNA modifications that improve siRNA selectivity, delivery and performance.

Gene Therapy (2006) 13, 509-516. doi:10.1038/sj.gt.3302726; published online 16 February 2006

Keywords: siRNA; single nucleotide polymorphisms; RISC assembly; Huntington's disease

\section{Introduction}

RNA interference (RNAi) provides therapeutic opportunity for many human diseases, but sometimes application of RNAi to specific disease genes is not apparent. Sequence constraints of a point mutation of a disease gene might limit siRNA design that distinguishes the mutant allele from the wild-type allele. Trinucleotide expansions in diseases such as Huntington's disease resist RNAi at the site of the mutation. The mutation is an expansion of CAG repeats from $=36$ to $>36 .{ }^{1}$ The small size of the siRNAs precludes their ability to distinguish mutant from wild-type huntingtin mRNAs. Are there strategic changes in siRNA design to improve siRNA efficacy? The goal of this review is to explain approaches of siRNA design to use in RNAi across a variety of genetic neurodegenerative diseases, based on current knowledge of mechanisms of siRNA action.

Essentials of RNAi can be summarized, to provide a reference for a detailed analysis. Small, double-stranded RNAs (about 21 nucleotides; siRNA) mediate destruction of a target mRNA. ${ }^{2,3} 5^{\prime}$ Phosphorylated siRNAs assemble with proteins to form a RNA-induced silencing complex (RISC). ${ }^{4}$ A single strand of the siRNA is selected to interact with the catalytic protein of RISC, Argonaute 2 (Ago 2). Formation of RISC precedes its binding to target mRNAs. Stabilized within a pocket in Ago 2, the guide strand of siRNA identifies the target mRNA and a catalytic domain of Ago 2 cleaves the mRNA at a vulnerable phosphate of the mRNA - determined by its position across from nucleotides 10 and 11 of the siRNA guide strand. Cleaved mRNA cannot be translated and its encoded protein is not produced. RISC dissociates from its target, to repeat this process; a few RISC molecules, therefore, can cleave many target mRNAs.

As with any therapeutics, the clinical usefulness of RNAi depends on its efficacy and safety. Thus, the

Correspondence: Professor N Aronin, University of Massachusetts, 55 Lake Avenue North, Worcester, MA 1655, USA.

E-mail: Aronin@umassmed.edu

Received 1 December 2005; revised 21 December 2005; accepted 5 January 2006; published online 16 February 2006 design of siRNAs should predict high effectiveness for destroying target mRNAs without affecting bystander mRNAs. To surmount some of these obstacles, siRNAs can be designed to recognize single nucleotide polymorphisms that, in theory, can be used to select mutant alleles for RNAi and leave wild-type allelic mRNA less perturbed. Understanding mechanisms of RISC assembly guides siRNA design, to improve RNAi efficacy. Here is limned a roadmap to explain RISC assembly how there are two types of RNAi, one of which is applicable to humans; how thermodynamic properties of siRNA direct strand selection to confer full RNAi activity; how RISC proteins direct siRNA presentation to its target mRNA; and how these principles can be used to design selective and functional siRNAs.

\section{Small RNAs start the RNAi process}

Innate mRNA silencing in mammals is currently the province of microRNAs, single stranded, small RNAs that block translation with short-term survival of target mRNA. In the past few years, the number of identified miRNAs in human has increased dramatically, from a few to estimates of nearly $1000 .^{5,6}$ siRNAs and miRNAs share protein associations in RISC; their precise activities become blurred and findings from miRNAs often apply to actions of siRNAs. A current distinction is that miRNAs derive from RNA transcripts containing a stem loop and load into RISC as single-stranded RNA. Often, miRNAs have nucleotide mismatches with targets. siRNAs begin as double-stranded RNA, need to be delivered to mammalian cells and require selection of the guide (antisense) strand from the passenger (sense) strand for incorporation into RISC. siRNAs in RNAi are the focus of this review.

\section{Mechanistic differences between RNAi in Caenorhabditis elegans and RNAi in Drosophila and vertebrates}

In a sense, there are two types of RNAi and the distinction between RNAi in nematodes and that in 
mammals allows the therapeutic potential for siRNA. RNAi in its original description in C. elegans is transitive. ${ }^{7}$ That is, the RNAi process spreads beyond the initial double-stranded RNA, or siRNA, stimulus. An RNAdependent RNA polymerase (RdRP) uses the target mRNA as a template to produce a new dsRNA, which then is processed to make more siRNAs. There is an increase in the number of siRNAs and the siRNAs target sites of mRNA beyond the initial site. This process is effective to eliminate an mRNA by targeting numerous sites in its sequence.

Transitive RNAi in mammals would render siteselective mRNA silencing difficult, if not impossible. Were it to pertain to mammals, introduction of a site selective siRNA would lead to generation of siRNAs that cut mRNA at multiple sites. The possibility of allelic discrimination would be eliminated. Thus, it is fortunate for RNAi therapeutics that transitive RNAi does not occur in mammals or in Drosophila, a model system applicable to RNAi mechanisms in mammals.

Much of our current knowledge of RNAi in mammals emanates from studies in Drosophila. To explore whether transitive RNAi pertains to Drosophila (and in mammals), alternative splicing for a gene was examined in cultured Drosophila cells. The gene contains 12 alternatively spliced mRNAs. ${ }^{8}$ If transitive RNAi were operative, then double-stranded, small RNA that targets one isoform would have destroyed many or all mRNA isoforms. However, the outcome shows selective RNAi; the alternatively spliced mRNAs remains intact. The implication of this experiment is that RNAi in Drosophila is not transitive, and double-stranded RNAs can be used to discriminate between similar mRNAs.

Another demonstration of the lack of transitive RNAi in Drosophila and mammals rests on the RdRP requirement for an unblocked $3^{\prime}$ end of siRNA. Blocking the $3^{\prime}$ end is known to eliminate RdRP activity. siRNA with blocked $3^{\prime}$ end was introduced into HeLa cells, without dampening silencing, ${ }^{9,10}$ indicating that RdRP priming is not needed in human cells. Roles for the $3^{\prime}$ hydroxyl and $5^{\prime}$ phosphate were studied in Drosophila and HeLa cells. ${ }^{10}$ Blocking phosphorylation of the $5^{\prime}$ end of the guide strand of the siRNA reduces RNAi efficacy. Substituting a hydroxyl there does not, because phosphorylation at the $5^{\prime}$ end occurs in cells. In contrast, blocking the $3^{\prime}$ end has little effect on RNAi. The implication of these results is that RNAi in mammals and Drosophila does not depend on RdRP and invokes cleavage of mRNA at a single site. Evidence for transitive RNAi is lacking in these cell types.

Another property of transitive RNAi is that mRNA silencing can spread to other tissues. In a Drosophila model for RNAi, transgenes containing dsRNA, which were localized to specific cells, has RNAi activity in the localized regions, with no evidence to spread to other tissues in the Drosophila. ${ }^{11}$ The RNAi was cell autonomous.

These findings confirm the possible usefulness of RNAi for therapeutics. siRNAs can be developed to detect specific mRNAs and silencing can be confined to cells that receive the siRNAs. Concerns about unintended knock down of mRNAs or imperfect delivery of siRNAs to tissues remain, but the main concern of transitive RNAi has been dispelled.

\section{Strand selection: thermodynamic properties of active siRNA}

In principle, successful implementation of RNAi would depend on careful design of the siRNA. Not all siRNAs are active, however, even when their guide strands have perfect complementarity to target mRNAs. Designing siRNAs with single nucleotide specificity requires the guide strand to be incorporated into RISC, in preference to the passenger strand. Thermodynamic profiling of siRNAs and biochemical experiments reveal unequal contributions of the placement of nucleotides in the guide strand. ${ }^{12-16}$ Examination of many siRNAs demonstrates that thermodynamic stability predicts RNAi activity. ${ }^{13}$ The strand of siRNA with the lower $5^{\prime}$ terminus thermodynamic stability is more functional. In study of 37 siRNAs for thermodynamic stability between strands, 16 of the siRNAs have $>70 \%$ knock down of target; 21 have less silencing activity. The lowest stability between the strands of the highly functional siRNAs is located at the $5^{\prime}$ terminus. In contrast, the less functional siRNAs are thermodynamically stable at the $5^{\prime}$ terminus. Furthermore, in the most functional siRNAs, the antisense (guide) strand is more unstable at the $5^{\prime}$ site compared to the sense (passenger) strand. Less functional siRNAs have $5^{\prime}$ terminal instability in the sense strand. The implication is that the energy profile of the siRNA determines its entry into RISC and its activity, rather than the match of the antisense strand to the mRNA target.

The idea that thermodynamic instability predicts strand selection was tested for two disease genes, SOD1 (hereditary amyotrophic lateral sclerosis) and $H D$ (Huntington's disease). ${ }^{14}$ In both situations, inactive siRNAs could be made functional by introducing mismatches (asymmetry) in the $5^{\prime}$ end of the siRNA guide. Conversely, thermodynamically stable siRNAs (symmetric) are not uniformly active, despite full complementarity of the guide strand to the target mRNA. Strand bias to enter RISC could explain these results. Thermodynamically less stable siRNAs would favor entry of either strand into RISC, whereas equal but high stability of the strands would favor neither. Therefore, strand selection can be predetermined by the purposive mismatch of nucleotides between the guide strand and the passenger strand.

\section{RISC formation}

Once the guide strand is assembled into a RISC, it is the RISC that cleaves the target mRNA. Two complementary approaches have established how siRNAs silence mRNA. Biochemical approaches unveiled components of the RISC and delineated steps to achieve assembly of siRNA and critical proteins. Double-stranded siRNA stabilizes its guide strand ${ }^{10}$ and, together with an endonuclease, ${ }^{17}$ cleaves mRNA reproducibly at the phosphate across from positions 10 and 11 of the guide strand (scissile phosphate). Crystallographic studies provide models that have been predicted by biochemical results and further explain the relationship between proteins of RISC (Ago 2) and the incorporated strand of the siRNA. ${ }^{18-22}$ 
siRNA therapeutics focuses on siRNA design, but it is how proteins, particularly Ago 2 in humans, incorporate the selected siRNA strand that underlies mRNA silencing. ${ }^{23}$ RISC proteins place the guide strand into a favorable position to bind to the target mRNA and juxtapose the catalytic unit of Ago 2 to a vulnerable phosphate bond across from nucleotides 10 and 11 of the guide strand. The complexity of siRNA design is that small changes in siRNA:mRNA complementarity can have profound effects on RNAi effectiveness; mismatches might have few consequences or, as shown above, improve silencing by strand selection.

$5^{\prime}$ Phosphorylation of the guide strand of siRNA is essential to the function of siRNA. This phosphorylation is one of the ATP dependent steps in RNAi. ${ }^{24}$ In Drosophila lysates, $5^{\prime}$ hydroxyl is rapidly phosphorylated in an ATP-dependent manner; substituting a $5^{\prime}$ group resistant to phosphorylation eliminates RNAi cleavage. A curiosity of the $5^{\prime}$ phosphorylation is that a matched, initial nucleotide is not required for RNAi function; ${ }^{14}$ crystallography later explained this observation.

The $5^{\prime}$ initial nucleotide determines the position of the critical endonuclease cleavage of the phosphate between nucleotides 10 and $11 .{ }^{25}$ The same site is cleaved whether the $3^{\prime}$ end of the guide strand of the siRNA varies to create a length 17, 19, or 21 nucleotides. Furthermore, several modifications of the $5^{\prime}$ end (2'-O-methyl) block RNAi, but modifications at the $3^{\prime}$ are tolerated. Human cells share the requirement of the $5^{\prime}$ phosphorylation. ${ }^{10}$

The $5^{\prime}$ end of the guide strand of the siRNA binds to the target mRNA; the $3^{\prime}$ end has a lesser role, if not negligible, in recognizing the mRNA to be cleaved. ${ }^{26}$ A detailed analysis of the $5^{\prime}$ end reveals that target recognition requires nucleotide complementarity at bases 2-5 of the guide strand, also known as the 'seed' sequence. ${ }^{26,27}$ However, it is the $3^{\prime}$ end that orients RISC and stabilizes the A-form of the newly formed helix (guide strand plus mRNA target). $3^{\prime}$ mismatches slow the rate of cleavage, but do not abolish cleavage. Up to nine mismatches at the $3^{\prime}$ terminus of siRNA will continue to cleave mRNA, albeit very slowly. ${ }^{27}$ Thus, the $5^{\prime}$ end of siRNA recognizes targeted mRNA, but lacks catalytic function; the $3^{\prime}$ end organizes the catalysis. Based on biochemical data, siRNA assembles with proteins, including an endonuclease; the proteins orient the siRNA to recognize and bind to a target mRNA, so that the endonuclease selectively cleaves the mRNA across from nucleotides 10 and 11 of the siRNA, at the scissile phosphate of the mRNA.

The endonuclease in RISC in mammals is Ago 2. ${ }^{28,29}$ A member of a family of Argonaute proteins that are capable of assembling with siRNA, only Ago 2 has catalytic function. Cells lacking Ago 2 have no RNAi. Single amino-acid substitutions in Ago 2 eliminate its endonuclease function. Ago 2 has two main structural domains: Piwi/Argonaute/Zwille (PAZ) and Piwi. The Piwi domain shares structural features of RNase $\mathrm{H}^{28}$

Double-stranded siRNA is thought to be more stable in cytoplasm than is single-stranded miRNA. However, a single-strand of the siRNA assembles into RISC, through placement into Ago 2. One strand is selected to insert into Ago 2. If thermodynamic equality pertains, the passenger strand can enter RISC and RNAi is reduced. The strand with relative thermodynamic instability is selected for the RISC-loading complex by R2D2. ${ }^{30,31}$
What happens to the rejected strand? At first ascribed to a helicase, separation of the two strands is now explained by destruction of one strand by Ago 2. ${ }^{32,33}$ In a fashion, the passenger strand is the initial target of the Ago 2 endonuclease activity. Blockade of passenger cleavage by either phosphorothioate ${ }^{32}$ or $2^{\prime}-O$-methyl ${ }^{33}$ modification of the passenger strand dampens RISC activity, but does not eliminate it. A cleavage-independent pathway is postulated, perhaps shared with the assembly mechanisms used in miRNA RISC formation. ${ }^{32}$ After cleavage, passenger strand remnants leave the RISC. The guide strand of the siRNA is secured and is no longer exchanged with free siRNAs. ${ }^{34}$ The RISC targets the mRNA, cleaves it, and dissociates to find another target. The human orthologous components of RISC, as set forth in Drosophila RNAi, form the essential RISC assembly in humans (human Dicer (Dcr2), TRBP (R2D2), hAgo 2 (Ago 2)). ${ }^{35}$ Combined with siRNA, these human orthologs effect RNAi in vitro. ${ }^{35}$ Figure 1 depicts a possible mechanism of RISC assembly.

Crystallographic studies of Ago 2 confirm and explain siRNA-Ago 2 interactions and RNAi activity. ${ }^{18,19}$ The tertiary structure of the Piwi domain of Ago 2 reveals $\mathrm{Mg}^{2+}$ stabilization of the structure $\left(\mathrm{Mg}^{2+}\right.$ dependence had been previously established) and structural similarity between Ago 2 and the RNase $\mathrm{H}$ endonuclease, which cleaves RNA-DNA heteroduplexes with DNA as the guide strand. The PAZ domain of Ago 2 does not interact with the $5^{\prime}$ end of siRNA that binds the target mRNA. The structure of Ago 2 predicts placement for both the siRNA guide strand and mRNA target. ${ }^{19}$ The presence of the divalent ion core and basic amino-acid residues nearby characterize structures that have phosphate binding. ${ }^{18}$ Placement of the $5^{\prime}$ phosphate of the siRNA into this site orients the catalytic unit of Ago 2 at a distance of $20 \AA$ from the scissile phosphate, conducive for endonuclease cleavage there. The siRNA interaction with Ago 2 stabilizes the seed sequence of the siRNA, especially nucleotides 2-5. Ago 2 positions the seed sequence to bind to mRNA and facilitates binding of siRNA nucleotides $6-8 .{ }^{21}$ Anchored by the extended seed sequence to the mRNA, Ago 2 is organized to cleave at the scissile phosphate. ${ }^{20,21}$ Human Ago 2 retains the same properties. $^{22}$ From crystallographic evidence, the $5^{\prime}$ phosphate of the guide strand confers activity of the siRNA by its positioning near the divalent stabilized site. Cleavage occurs at predictable distances from the $5^{\prime}$ phosphate, the scissile phosphate. The $5^{\prime}$ end of the guide strand of the siRNA binds the mRNA, after it is oriented within the Ago 2. RISC releases the target mRNA after cleavage, but Ago 2 alone does not, implicating roles for other proteins in RISC.

\section{Designing siRNAs for therapy}

Optimal siRNAs favor the guide strand entering Ago 2 to the exclusion of the passenger strand, thereby directing RISC to bind to the target mRNA, where potent cleavage of the target ensues. For effective RNAi, the $5^{\prime}$ end of the guide strand requires sufficient complementarity to bind to the target and the orientation of the $3^{\prime}$ end of the guide strand needs to favor catalysis of the target by Ago 2. Nonspecific cleavage of mRNA - unintended mRNA targets, or 'off-targets' - would be undesirable in RNAi 
therapeutics. mRNA targets in hereditary diseases or somatic gene mutations might differ from wild-type mRNA by a single nucleotide change. Single nucleotide polymorphisms offer another potential site for RNAi

$R L C$
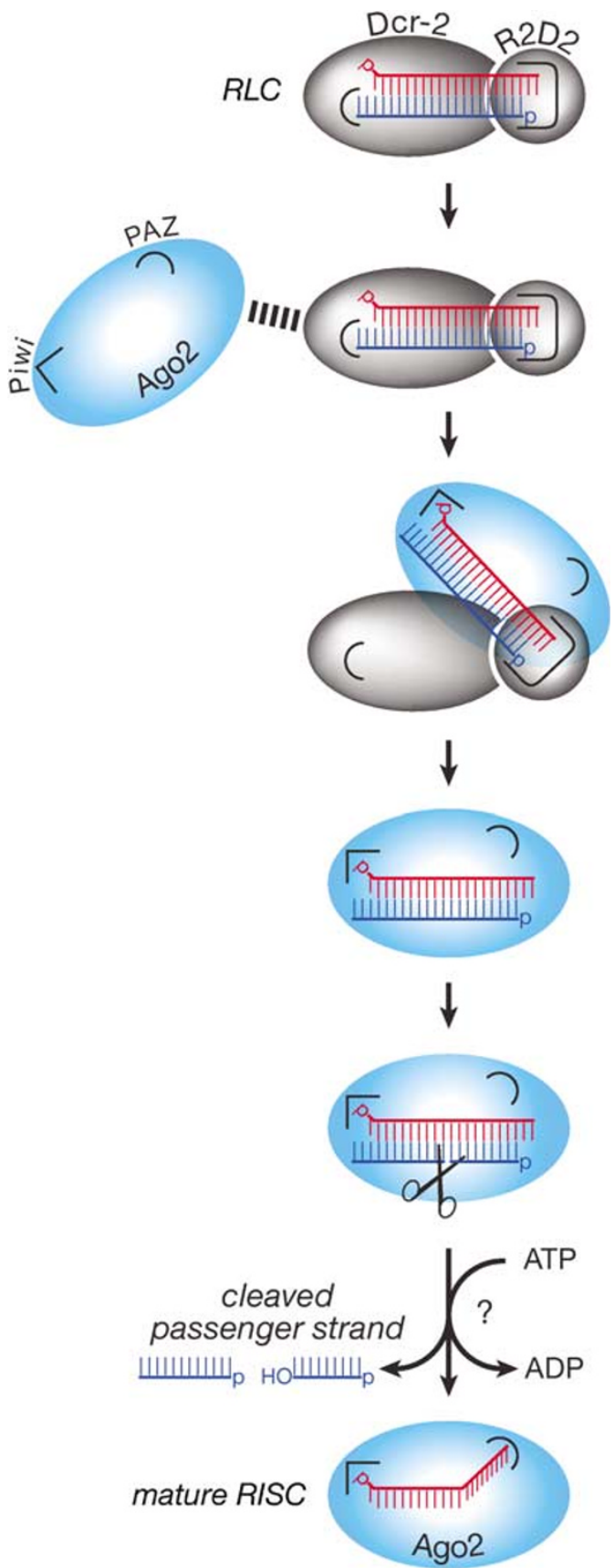

Figure 1 A revised model for RISC assembly. This model depicts RNAi events from data derived in study of RNAi in Drosophila, but it is anticipated that similar events apply to mammalian RNAi. Dcr2 and R2D2 recruit Ago 2 to the double-stranded siRNA, to form a RISC Loading Complex (RLC). Ago 2 exchanges with Dcr-2 by a protein-protein contact (dashed line) ${ }^{36}$ and then exchanges with R2D2. Ago 2 endonuclease activity cleaves the passenger strand (blue). The guide strand (red) separates from the siRNA duplex and produces a mature, active RISC. Release of the passenger-strand cleavage products may be facilitated by an ATP-dependent cofactor, much as release of the products of target cleavage facilitated by ATP. ${ }^{27}$ The mature RISC is now capable for executing RNAi to a target mRNA. Adapted with permission from Matranga et al. ${ }^{32}$ therapy. Therefore, siRNA designs will need to account for such small sequence changes. Furthermore, in general, mammalian cells resist uptake of doublestranded RNA, thereby requiring formulations or conjugations of siRNAs to facilitate their cellular uptake.

Maximizing guide strand selection is an initial, critical goal in siRNA design. Based on the previously discussed thermodynamic properties of asymmetric siRNAs, ${ }^{9-11}$ guide strands of siRNA can be predictably recruited into RISC. Inactive siRNAs, or less than optimally effective siRNAs, can achieve better RNAi by introducing nucleotide mismatches (thermodynamically unstable nucleotide pairings). An example for huntingtin mRNA is provided (Figure 2). Strategically placed nucleotide mismatches can increase the RNAi by the guide strand, as noted by the increase in cleaving its target of huntingtin mRNA. Improved RNAi activity would reduce therapeutic dose requirements, because more of the active guide strand than passenger strand enters RISC. Passenger strand effects have been implicated to have off-target effects; limiting passenger strand loading into RISC mitigates this concern. Use of asymmetric siRNA can be extended to design shRNAs for viral delivery of siRNA.

A next goal in siRNA design is optimizing its single nucleotide discrimination. This approach is useful for RNAi at point mutations and single nucleotide polymorphisms (SNP), allowing RNAi application to distinguish between a mutant allele and its wild-type allele for autosomal dominant diseases dependent on a single
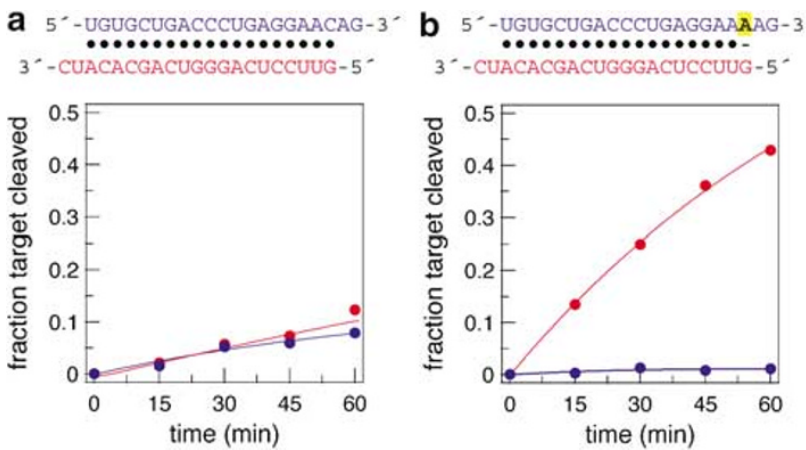

Figure 2 Use of rules of siRNA asymmetry for RNAi of huntingtin mRNA. Incorporation of the guide strand into RISC determines the RNAi activity of a siRNA. In the experiment shown here, the blue strand is the passenger in the siRNA and the red strand is the guide. (a and $\mathbf{b}$ ) The ability of the guide strand (in red) to effect RNAi is tested against a synthetic huntingtin mRNA target (in red in figure). The RNAi activity of the passenger strand (in blue) is tested against an artificial mRNA target to which it has complementarity (in blue in figure). (a) Both the guide strand and the passenger strand have equal potency in cleaving their targets. This result indicates that both guide and passenger strands in a huntingtin siRNA can enter RISC and cleave a mRNA with complementarity to a siRNA strand. (b) The huntingtin siRNA has a nucleotide substitution (purple A, highlighted yellow) near the $3^{\prime}$ end of the passenger strand. This nucleotide is a purposeful mismatch, creating an asymmetry in the siRNA. A consequence of the asymmetry is that the guide strand is preferred to enter RISC. The huntingtin mRNA is cleaved and the artificial mRNA with complementarity to the passenger strand is not cleaved. Therefore, asymmetric siRNAs can improve the efficacy of the siRNA in mRNA silencing, because they direct the guide (antisense) strand into RISC. Adapted with permission from Schwarz et al..$^{14}$ 
point mutation. The same principle can be invoked for nucleotide differences in oncogenes and proto-oncogenes. Single nucleotide recognition by siRNA has been demonstrated for siRNAs directed against sod1 (gene for hereditary amyotrophic lateral sclerosis); ${ }^{37}$ and a missense Tau mutation that results in dementia. ${ }^{38}$ Single nucleotide discrimination, in theory, allows RNAi to be applied to single nucleotide polymorphisms (SNP), to parse allelic targets. Diseases caused by expansion of trinucleotide repeats, such as the increased CAG repeat number in Huntington's disease, would ordinarily be resistant to mRNA silencing. siRNAs are about 21 nucleotides in length, too short to distinguish between the wild type and mutant huntingtin mRNA based solely on CAG repeat number. However, RNAi can be useful to recognize SNPs, as shown for knock down of MJD1 (Machado-Joseph Disease). ${ }^{31}$ A caveat for single nucleotide discrimination is its position in the siRNA. Since Ago 2 positions the seed sequence of the siRNA to bind to mRNA, a mismatch in bases $2-5$ in the $5^{\prime}$ end of the siRNA might reduce siRNA binding. Mismatches in nucleotides in the $3^{\prime}$, catalytic end of the guide strand might alter cleavage of the scissile phosphate. ${ }^{22,23,27}$ The nucleotide mismatch(es) should either abrogate binding or cleavage of the siRNA to the wildtype mRNA, while maintaining full activity to the mutant, target mRNA.

It is predicted that some nucleotide mismatches might be tolerated and retain RNAi activity. The A G:U wobble between the guide strand and the target mRNA has a negative free energy and preserves partial complementarity. In contrast, a purine:purine mismatch between the guide strand and the target mRNA has an unfavorable free energy, lacks complementarity and would hinder RISC binding and therefore RNAi. These ideas have begun to be probed. In study of a small set of siRNAs and targets, mismatched nucleotide pairs at the ends of siRNA conserve RNAi activity and are better tolerated compared to central mismatches. ${ }^{39}$ Certain nucleotide mismatches were compatible with RNAi. A:C and G:U mismatches did not nullify RNAi. However, other mismatches - A:A, A:G, and $U: U$ - reduced RNAi activity; the extent of the reduction varied with the position of the mismatch. A hierarchy of position and nucleotide mismatch in RNAi effectiveness has not yet established. It is proposed that internal nucleotide mismatches of a guide strand with target mRNA are likely to yield discrimination of siRNA at single base sites. For selective RNAi of an allele with a mutation and preservation of the wild-type allele, each SNP site might need to be identified and tested for allelic RNAi discrimination.

Additional mismatches at non-SNP sites might offer additional discrimination between wild type and disease alleles. $^{38}$ Since formation of RISC does not require full complementarity with target mRNA, it is possible that an internal nucleotide mismatch of the guide strand against the target mRNA might be tolerated, but the presence of two mismatches (at a SNP and one other) against a wildtype mRNA would attenuate or eliminate its RNAi activity. Creative siRNA design will take advantage of properties of binding and catalytic functions of siRNA, the relative tolerance of nucleotide mismatches, and sequence differences between targeted and nontargeted mRNAs.
Not all mRNAs are amenable to RNAi. Accessibility of siRNA to its target might improve effectiveness of silencing in recalcitrant mRNAs. RNAi of HIV-1 transactivation responsive element (TAR) provides an example of how improving access to the mRNA increases RNAi efficacy. ${ }^{40}$ TAR RNA has a stem loop structure, resistant to conventional RNAi. Addition of 20 nucleotide $2^{\prime}-O$-methyl oligonucleotides either $5^{\prime}$ or $3^{\prime}$ of the TAR siRNA binding site improves cleavage of the TAR RNA, from 2 to $65 \%$. It is speculated that the oligonucleotide clamps stabilize the target RNA, facilitating RISC access to its target and subsequent RNAi.

Gene products in pathogenic pathways can be useful targets in RNAi therapy for neurodegenerative diseases. A pathogenic mechanism in Alzheimer's disease is the accumulation of an enzymatic cleavage fragment of amyloid precursor protein, amyloid- $\beta$ protein. The enzyme responsible for this cleavage is $\beta$-secretase; RNAi against $\beta$-secretase in an in vivo model of Alzheimer's disease reduces amyloid deposition in the hippocampus and improves spatial learning in a Morris water-maze. $^{41}$ The salubrious effects of the RNAi are found with reduction of $\beta$-secretase; elimination of the enzyme is not needed and may not be sought because of its multiple cellular functions.

\section{Potential risks of RNAi}

Recent studies have highlighted potential side effects of RNAi: its possible off target effects. In certain experimental conditions, RNAi changes the expression profiles of unintended mRNAs. ${ }^{42-46}$ mRNA silencing from off target effects can result in RNAi (destruction of the off target mRNA) or translational repression (survival of mRNA in the short-term) ${ }^{45}$ Mismatches of up to four nucleotides and G:U wobbles can lead to translational repression of candidate off target mRNAs. Further examination of off target silencing has made the surprising observation that the transfectant used for siRNA has its own effects on expression profiles, which are separate from the siRNA itself. ${ }^{47}$ Further studies in vivo should help to resolve the extent of off target siRNA effects. Lacking in studies of nonspecific mRNA silencing is the protein profile of the off target mRNAs. A small change in mRNA expression does not necessarily determine a biologically important change in its cognate protein. Minimal, but reproducible, changes in protein profiles might have clinical significance. Forecasting specific untoward effects based on subtle changes in mRNA content at this point is a leap of faith.

Another unresolved question is the extent to which siRNA stimulates induction of cellular immunomodulatory responses. A clear answer has not been achieved. siRNAs have been shown to provoke an interferon response. ${ }^{43}$ Toll-3 receptors recognize double-stranded RNA and can mediate activation of NF- $\kappa \mathrm{B}$, which regulates interleukin responses, ${ }^{48}$ providing a substrate for siRNA immune-activation. However, naked siRNAs injected into mouse tail vein do not elicit an interferon a or IL-12 response. ${ }^{49}$ Long poly I:C induces increased interferon, but predigested small strands of poly I:C do not. The implication is that siRNAs are sufficiently small to escape an immune provocation. In contrast, systemic injection of siRNA packaged in liposomes increases the 
inflammatory cytokines interleukin- 6 and tumor necrosis factor $\alpha .{ }^{50}$ siRNAs administered with liposomes generate increase release of these cytokines in vitro in human blood. Interleukin-6 and tumor necrosis factor-a show no change after administration of unmodified, naked siRNA at the same dose. Although most siRNAs delivered in liposomes elicit an increase in cytokines, nucleotide substitution in siRNA mitigates or abolishes the inflammatory response. Three siRNAs have been studied, so that it is difficult to generalize the findings to siRNA design. Nonetheless, the observation supports the idea that siRNA influences on inflammatory responses depend on contemporaneous administration of vehicles and inherent immunomodulatory properties of the siRNA sequence. A daunting outcome is raised, that each siRNA formulation might have its own risks.

The mode of delivery might influence the nature of the immune response. siRNA delivered intranasally to mice and primates does not stimulate immunomodulatory responses. siRNA against respiratory syncytial virus does not increase interferon in lung, ,51,52 although it effectively reduces lung pathology when administered before, with and after introduction of the virus. In a Rhesus monkey model of SARS, nasally administered siRNA against the SARS coronavirus effectively reduces the viral infection and lung pathology. ${ }^{53}$ An observed increase in liver enzymes is no greater after SARS infection with siRNA than without siRNA.

Improving bioavailability of siRNA, thereby reducing its required dose, could theoretically attenuate toxicities. Incorporation of locked nucleic acids (LNA) into siRNAs increases their stability in serum. ${ }^{54}$ Unmodified siRNA in plasma is degraded in $6 \mathrm{~h}$, but LNA modified siRNAs last over $48 \mathrm{~h}$. The LNA modified siRNA maintained RNAi activity in vitro, except at position 10, which alters the A-form structure required for RNAi. Toxicity of LNA modified siRNAs will need further testing in vivo.

Introduction of siRNA into cells raises a theoretical concern: might exogenous siRNA compete with endogenous, intracellular mRNA silencing systems? It is now established that mammalian cells have many miRNAs, perhaps one thousand, ${ }^{6}$ which in principle share proteins that form RISC. ${ }^{4}$ The stoichiometry of proteins such as Ago 2 and R2D2 in relationship to miRNAs is not yet known. Flooding a cell with siRNA might prevent miRNAs from participating in mRNA silencing. The same provision applies to viral delivery of shRNAs. This possible risk highlights the importance of using the most selective siRNA at the lowest therapeutic dose.

\section{Delivery}

Getting siRNAs into cells in vivo has developed into a formidable barrier for siRNA therapy. Viral delivery of short hairpin RNA (shRNA) has been shown to effect RNAi in models of neurodegenerative disease, SCA1, ${ }^{55}$ HD, ${ }^{56,57}$ and ALS. ${ }^{5,59}$ High doses of naked siRNA have been effective; intrathecal administration of naked siRNA $(400 \mu \mathrm{g} /$ day for 1 week) against mRNA for a pain-related cation channel, P2X3, lessened acute and chronic pain responses in rat. ${ }^{60}$ Intraventricular administration of naked siRNA for 1 week decreased mRNA and protein for a dopamine transporter in mice. ${ }^{61}$ Known as the hydrodynamic approach, administration of naked
siRNA in large volumes of saline in mice can reduce target mRNA, ${ }^{62}$ exemplified in the abrogation of acute, severe hepatitis. ${ }^{63}$ The hydrodynamic approach causes right heart failure in mice and would be inappropriate for human use.

Modifications of siRNA to improve its in vivo bioavailability offer encouraging results; the nucleotide substitutes are chosen to reduce digestion of siRNA. In addition to the theoretical use of locked nucleic acid substitutions in siRNA, ${ }^{54}$ substituting $2^{\prime} \mathrm{F}, 2^{\prime} \mathrm{O}-\mathrm{Me}$, or $2^{\prime} \mathrm{H}$ residues for all $2^{\prime}-\mathrm{OH}$ residues stabilizes siRNAs that were used to treat hepatitis B virus in a mouse model. ${ }^{64}$ The siRNAs are administered in a specialized liposome to mice. siRNAs are designed to recognize HBV. Robust reduction in HBV serum DNA lasts 7 days after initial administrations of siRNA; a lesser reduction of $\mathrm{HBV}$ persists for 6 weeks.

Conjugation of siRNA to carrier molecules presents a novel strategy to improve cellular delivery. Mechanisms of siRNA entry into cells are not yet established. Chemical additions include peptides, such as TAT (4757), ${ }^{65}$ and Penetratin1. ${ }^{66}$ The delivery of Penetratin1conjugated siRNA against sod1 and caspase 3 in neurons has the unexpected finding that protein reduction preceded a reduction in their related mRNAs. The implication is that - under these experimental conditions - the conjugated siRNAs invoke translational repression.

Administration of cholesterol conjugated siRNA against $a p o B$, a constituent of low-density lipoproteins, into mouse circulation has good potency in knocking down hepatic $a p o B$ and reducing serum concentrations of low-density lipoproteins. ${ }^{67}$ Cholesterol conjugation of siRNA improves its stability in serum incubation. Unconjugated apoB-1-siRNA lacks a biological effect in vivo. Whether the addition of cholesterol improves transport of the siRNA across plasma membranes or serves as ligand for cholesterol related, receptormediated endocytosis is not established.

Cell selectivity remains a formidable obstacle. Improving specific cellular uptake of siRNA not only favors RNAi for target mRNAs in appropriate settings, but also makes available siRNA for specific cells. In theory, those cells that a siRNA recognizes will preferentially take up the siRNA. Conjugation of Fab antibody to siRNA provides evidence that cell selectivity is feasible. siRNA attached through strong ionic bonds to an antibodyprotamine fusion protein. ${ }^{68}$ The siRNA is directed against HIV-1 env and the antibody is directed against HIV envelope. HIV envelope is expressed in T lymphocytes or melanoma cells in vivo. Infusion of the antibody tagged siRNA inhibits HIV replication under both conditions. This study indicates that siRNAs can be directed to specific cell surface proteins and taken up into the target cells, for RNAi.

Delivery of siRNAs to effect treatment is a surmountable hurdle. Preclinical studies have shown successful delivery of siRNA by inhalation and intravenous injection. Delivery can be local, systemic, and cell selective. Whether conjugations that enhance delivery interfere with siRNA incorporation into RISC will need to be explored. It is conceivable that alteration of the $5^{\prime}$ insertion of the guide strand into Ago 2 could mitigate RNAi activity and lead to the need for higher doses of siRNA. Nonetheless, rapid advances in knowledge of siRNA design foretell that this theoretical concern will be solved, too. 


\section{Summary}

In its most basic terms, siRNAs can be designed to have full complementarity to a target mRNA. With good fortune, the siRNA will invoke RNAi. Designing siRNAs for gene therapy adds complexity: not all siRNAs are effective, not all disease genes are amenable to RNAi at the disease-related mRNA sequence, caveats - off target and interferon responses - attend RNAi, and cells in vivo might shun siRNA. Knowledge of mechanisms in RNAi and innovations in siRNA delivery offer promise for its use in gene therapy. A pivotal point in mRNA silencing is the selection of the active strand of the siRNA duplex. Nucleotide modifications can bias incorporation of the most active strand into RISC. Understanding this process should improve siRNA activity and allele selectivity. Sequences of siRNAs have functional importance, for association with the target mRNA, its initial cleavage at vulnerable sites and its overall catalytic action. In theory, changes in sequence of the siRNA can improve single nucleotide dependent RNAi, with little effect on RISC endonuclease activity. The therapeutic implication is allele specificity, to knock down disease linked mRNA while retaining the wild-type allele. How clinically relevant are off target and inflammatory responses remains to be established. Therapy with siRNAs has become tractable.

\section{Acknowledgements}

I would like to thank Dr Phillip Zamore and Dr Dianne Schwarz for their helpful suggestions.

\section{References}

1 Huntington's Disease Collaborative Research Group. A novel gene containing a trinucleotide repeat that is unstable on Huntington's disease chromosomes. Cell 1993; 72: 971-983.

2 Tuschl T, Zamore PD, Lehmann R, Bartel DP, Sharp PA. Targeted mRNA degradation by double-stranded RNA in vitro. Genes Dev 1999; 13: 3191-3197.

3 Tomari Y, Zamore PD. Perspective: machines for RNAi. Genes Dev 2005; 19: 517-529.

4 Zamore PD, Tuschl T, Sharp PA, Bartel DP. RNAi: doublestranded RNA directs the ATP-dependent cleavage of mRNA at 21-23 nucleotide intervals. Cell 2000; 101: 25-33.

5 Berezikov E, Guryev V, van de Belt J, Wienholds E, Plasterk RH, Cuppen E. Phylogenetic shadowing and computational identification of human microRNA genes. Cell 2005; 120: 21-24.

6 Lewis BP, Burge CB, Bartel DP. Conserved seed pairing, often flanked by adenosines, indicates that thousands of human genes are microRNA targets. Cell 2005; 120: 15-20.

7 Fire A, Xu S, Montgomery MK, Kostas SA, Driver SE, Mello CC. Potent and specific genetic interference by double-stranded RNA in Caenorhabditis elegans. Nature 1998; 391: 806-811.

8 Celotto AM, Graveley BR. Exon-specific RNAi: a tool for dissecting the functional relevance of alternative splicing. RNA 2002; 8: 718-724.

9 Chiu YL, Rana TM. RNAi in human cells: Basic structural and functional features of small interfering RNA. Molec Cell 2002; 10: 549-561.

10 Schwarz DS, Hutvagner G, Haley B, Zamore PD. Evidence that siRNAs function as guides, not primers, in the Drosophila and human RNAi pathways. Mol Cell 2002; 10: 537-548.
11 Roignant J-Y, Carre C, Mugat B, Szymczak D, Lepesant J-A, Antoniewsi $C$. Absence of transitive and systemic pathways allows cell-specific and isoform-specific RNAi in Drosophila. RNA 2003; 9: 299-308.

12 Aza-Blanc P, Cooper CL, Wagner K, Batalov S, Deveraux QL, Cooke MP. Identification of modulators of TRAIL-induced apoptosis via RNAi-based phenotypic screening. Mol Cell 2003; 12: 627-637.

13 Khvorova A, Reynolds A, Jayasena SD. Functional siRNAs and miRNAs exhibit strand bias. Cell 2003; 115: 209-216.

14 Schwarz DS, Hutvagner G, Du T, Xu Z, Aronin N, Zamore PD. Asymmetry in the assembly of the RNAi enzyme complex. Cell 2003; 115: 199-208.

15 Reynolds A, Leake D, Boese Q, Scaringe S, Marshall WS Khvorova A. Rational siRNA design for RNA interference. Nat Biotechnol 2004; 22: 326-330.

16 Ui-Tei K, Naito Y, Takahashi F, Haraguchi T, Ohki-Hamazaki H, Juni A et al. Guidelines for the selection of highly effective siRNA sequences for mammalian and chick RNA interference. Nucleic Acids Res 2004; 32: 936-948.

17 Hammond SM, Bernstein E, Beach D, Hannon GJ. An RNAdirected nuclease mediates post-transcription gene silencing in Drosophila cells. Nature 2000; 404: 293-296.

18 Parker JS, Roe SM, Barford D. Crystal structure of a PIWI protein suggests mechanisms for siRNA recognition and slicer activity. EMBO J 2004; 23: 4727-4737.

19 Song JJ, Smith SK, Hannon GJ, Joshua-Tor L. Crystal structure of Argonaute and its implication for RISC slicer activity. Science 2004; 305: 1434-1437.

20 Parker JS, Roe SM, Barford D. Structural insights into mRNA recognition from a PIWI domain-siRNA guide complex. Nature 2005; 434: 663-666.

21 Ma JB, Yuan YR, Meister G, Pei Y, Tuschl T, Patel DJ. Structural basis for $5^{\prime}$-end-specific recognition of guide RNA by the $A$. fulgidus Piwi protein. Nature 2005; 434: 666-670.

22 Rivas FV, Tolia NH, Song JJ, Aragon JP, Liu J, Hannon GJ et al. Purified Argonaute 2 and an siRNA form recombinant human RISC. Nat Struct Mol Biol 2005; 12: 340-349.

23 Hammond SM, Boettcher S, Caudy AA, Kobayashi R, Hannon GJ. Argonaute2, a link between genetic and biochemical analyses of RNAi. Science 2001; 293: 1146-1150.

24 Nykanen A, Haley B, Zamore PD. ATP requirements and small interfering RNA structure in the RNA interference pathway. Cell 2001; 107: 309-321.

25 Elbashir SM, Martinez J, Patkaniowska A, Lendeckel W, Tuschl T. Functional anatomy of siRNAs for mediating efficient RNAi in Drosophia melanogaster embryo lysate. EMBO J 2001; 20: 6877-6888.

26 Doench JG, Sharp PA. Specificity of microRNA target selection in translational repression. Genes Dev 2004; 18: 504-511.

27 Haley B, Zamore PD. Kinetic analysis of the RNAi enzyme complex. Nat Struct Mol Biol 2004; 11: 599-606.

28 Liu J, Carmell MA, Rivas FV, Marsden CG, Ghomson JM, Song JJ et al. Argonaute2 is the catalytic engine of mammalian RNAi. Science 2004; 305: 1437-1441.

29 Meister G, Landthaler M, Patkaniowska A, Dorsett Y, Teng G, Tuschl T. Human Argonaute2 mediates RNA cleavage targeted by miRNAs and siRNAs. Mol Cell 2004; 15: 185-197.

30 Tomari Y, Matranga C, Haley B, Martinez N, Zamore PD. A protein sensor for siRNA asymmetry. Science 2004; 306: 1377-1380.

31 Liu Q, Rand TA, Kalidas S, Du F, Kim HE, Smith DP et al. R2D2, a bridge between the initiation and effector steps of the Drosophila RNAi pathway. Science 2003; 301: 1921-1925.

32 Matranga C, Tomari Y, Shin C, Bartel DP, Zamore PD. Passengerstrand cleavage facilitates assembly of siRNA into Ago 2-containing RNAi enzyme complexes. Cell 2005; 123: 607-620. 
33 Rand T, Petersen S, Du F, Wang X. Argonaute2 cleaves the antiguide strand of siRNA during RISC activation. Cell 2005; 123: 621-629.

34 Martinez J, Patkaniowska A, Urlaub H, Luhrmann R, Tuschl T. Single-stranded antisense siRNAs guide target RNA cleavage in RNAi. Cell 2002; 110: 563-574.

35 Gregory RI, Chendrimada TP, Cooch N, Shiekhattar R. Human RISC couples microRNA biogenesis and posttranscriptional gene silencing. Cell 2005; 123: 631-640.

36 Tahbaz N, Kolb FA, Zhang H, Jaronczyk K, Filipowicz W, Hobman TC. Characterization of the interactions between mammalian PAZ PIWI domain proteins and Dicer. EMBO Rep 2004; 5: 189-194.

37 Ding H, Schwarz DS, Keene A, Affar el B, Fenton L, Xia X et al. Selective silencing by RNAi of a dominant allele that causes amyotrophic lateral sclerosis. Aging Cell 2003; 2: 209-217.

38 Miller VM, Xia H, Marrs GL, Gouvion CM, Lee G, Davidson BL et al. Allele-specific silencing of dominant disease genes. Proc Natl Acad Sci 2003; 100: 7195-7200.

$39 \mathrm{Du}$ Q, Thonberg H, Wang J, Wahlestedt C, Liang Z. A systemic analysis of the silencing effects of an active siRNA at all singlenucleotide mismatched target sites. Nucleic Acids Res 2005; 33: 1671-1677.

40 Brown KM, Chu C-Y, Rana TM. Target accessibility dictates the potency of human RISC. Nat Struct Mol Biol 2005; 12: 469-470.

41 Singer O, Marr RA, Rockenstein E, Crews L, Coufal NG, Gage FH et al. Targeting BACE1 with siRNAs ameliorates Alzheimer disease neuropathology in a transgenic model. Nat Neurosci 2005; 8: 1343-1349.

42 Jackson AL, Bartz SR, Schelter J, Kobayashi SV, Burchard J, Mao $\mathrm{M}$ et al. Expression profiling reveals off-target gene regulation by RNAi. Nat Biotechnol 2003; 21: 635-637.

43 Sledz CA, Holko M, de Veer MJ, Silverman RH, Williams BR. Activation of the interferon system by short-interfering RNAs. Nat Cell Biol 2003; 5: 834-839.

44 Scacheri PC, Rozenblatt-Rosen O, Caplen NJ, Wolfsberg TG, Umayam L, Lee JC et al. Short interfering RNAs can induce unexpected and divergent changes in the levels of untargeted proteins in mammalian cells. Proc Natl Acad Sci USA 2004; 101: 1892-1897.

45 Persengiev SP, Zhu X, Green MR. Nonspecific, concentrationdependent stimulation and repression of mammalian gene expression by small interfering RNAs (siRNAs). RNA 2004; 10: 12-18.

46 Saxena S, Jonsson ZO, Dutta A. Small RNAs with imperfect match to endogenous mRNA repress translation: Implications for off-target activity of siRNA in mammalian cells. J Biol Chem 2003; 278: 444312-444319.

47 Federov Y, King A, Anderson E, Karpilow J, Ilsley D, Marshall W et al. Different delivery methods-different expression profiles. Nat Methods 2005; 2: 241.

48 Alexopoulou L, Holt AC, Medzhitov R, Flavell RA. Recognition of double-stranded RNA and activation of NF- $\kappa$ B by Toll-like receptor 3. Nature 2001; 413: 732-738.

49 Heidel JD, Hu S, Liu XF, Triche TJ, Davis ME. Lack of interferon response in animals to naked siRNAs. Nat Biotechnol 2004; 22: 1579-1582.

50 Judge AD, Sood V, Shaw JR, Fang D, McClintock K, Maclachlan I. Sequence-dependent stimulation of the mammalian innate immune response by synthetic siRNA. Nat Biotechnol 2005; 23: 457-462.

51 Bitko V, Musiyenko A, Shulyayeva O, Barik S. Inhibition of respiratory viruses by nasally administered siRNA. Nat Med 2005; 11: 5-55.
52 Zhang W, Yang H, Kong X, Mohapatra S, San Juan-Vergara H, Hellermann $G$ et al. Inhibition of respiratory syncytial virus infection with intranasal siRNA nanoparticles targeting the viral NS1 gene. Nat Med 2005; 11: 56-62.

53 Li BJ, Tang Q, Cheng Du, Qin C, Xie FY, Wei Q et al. Using siRNA in prophylactic and therapeutic regimens against SARS coronavirus in Rhesus macaque. Nat Med 2005; 11: 944-951.

54 Elman J, Thonberg H, Ljunberg K, Frieden M, Westergaard M, $\mathrm{Xu} \mathrm{Y}$ et al. Locked nucleic acid (LNA) mediated improvements in siRNA stability and functionality. Nucleic Acids Res 2005; 33: 439-447.

55 Xia H, Mao Q, Eliason SL, Harper SQ, Martins IH, Orr HT et al. RNAi suppresses polyglutamine-induced neurodegeneration in a model of spinocerebellar ataxia. Nat Med 2004; 10: 816-820.

56 Harper SZ, Staber PD, He X, Eliason SL, Martins IH, Mao Q et al. RNA interference improves motor and neuropathological abnormalities in a Huntington's disease mouse model. Proc Natl Acad Sci 2005; 102: 5820-5825.

57 Rodriguez-Lebron E, Denovan-Wright EM, Nash K, Lewin AS, Mandel RJ. Intrastriatal rAAV-mediated delivery of anti-huntingtin shRNAs reduces partial reversal of disease progression in R6/1 Huntington's disease transgenic mice. Mol Ther 2005; 12: 618-633.

58 Raoul C, Abbas-Terki T, Bensadoun JC, Guillot S, Haase G, Szulc $\mathrm{J}$ et al. Lentiviral-mediated silencing of SOD1 through RNA interference retards disease onset and progression in a mouse model of ALS. Nat Med 2005; 11: 423-428.

59 Ralph GS, Radcliffe PA, Day DM, Carthy JM, Leroux MA, Lee DCP et al. Silencing mutant SOD1 using RNAi protects against neurodegeneration and extends survival in an ALS model. Nat Med 2005; 11: 429-433.

60 Dorn G, Patel S, Wotherspoon G, Hemmings-Mieszczak M, Barclay J, Natt FJC et al. siRNA relieves chronic neuropathic pain. Nucl Acids Res 2004; 32: e49.

61 Thakker DR, Natt F, Husken D, Maier R, Muller M, van der Putten $\mathrm{H}$ et al. Neurochemical and behavioral consequences of widespread gene knockdown in the adult mouse brain using nonviral RNA interference. Proc Natl Acad Sci 2004; 101: 17270-17275.

62 Lewis DL, Wolff JA. Delivery of siRNA and siRNA expression constructs to adult mammals by hydrodynamic intravascular injection. Methods Enzymol 2005; 392: 336-350.

63 Song E, Lee SK, Wang J, Ince N, Ouyang N, Min J et al. RNA interference targeting Fas protects mice from fulminant hepatitis. Nat Med 2003; 9: 347-351.

64 Morrissey DV, Lockridge JA, Shaw L, Blanchard K, Jensen K, Breen $\mathrm{W}$ et al. Potent and persistent in vivo anti-HBV activity of chemically modified siRNAs. Nat Biotechnol 2005; 23: 1002-1007.

65 Chiu YL, Rana TM. siRNA function in RNAi: a chemical modification analysis. RNA 2003; 9: 1034-1048.

66 Davidson TJ, Harel S, Arboleda VA, Prunell GF, Shelanski ML, Greene LA et al. Highly efficient small interfering RNA delivery to primary mammalian neurons induces MicroRNA-like effects before mRNA degradation. J Neurosci 2004; 24: 10040-10046.

67 Soutschek J, Akinc A, Branlage B, Charisse K, Constien R, Donoghue $\mathrm{M}$ et al. Therapeutic silencing of an endogenous gene by systemic administration of modified siRNAs. Nature 2004; 432: 173-178.

68 Song E, Zhu P, Lee S-K, Chowdhury D, Kussman S, Dykxhoorn DM et al. Antibody mediated in vivo delivery of small interfering RNAs via cell-surface receptors. Nat Biotechnol 2005; 23: 709-717. 